\title{
XÁC ĐİNH ĐĂC TRƯNG ĐÀN HỒI CỦA TẤM VÀ ỐNG VẬT LIỆU CẤU TRÚC NA NÔ BẰNG PHƯƠNG PHÁP PHẦN TỦ HŨUU HẠN NGUYÊN TỬ
}

\author{
Nguyễn Danh Trường ${ }^{*}$, Lê Minh Quý \\ Viện Cơ khí, Trường Đại học Bách khoa Hà Nội, Số 1 Đại Cồ Việt, Hà Nội \\ "Email: truong.nguyendanh@hust.edu.vn
}

Đến Tòa soạn: 12/06/2014; Chấp nhận đăng: 5/3/2015

\section{TÓM TẮT}

Phương pháp phần tử hữu hạn nguyên tử (AFEM) được phát triển để xác định đặc trưng đàn hồi của ống các bon na nô đơn lớp, ống boron nitride (BN), tấm $\mathrm{BN}$ và graphen. Sử dụng cùng các hằng số lực, kết quả mô đun đàn hồi khi tính bàng AFEM sai khác so với kết quả tính bằng động lực phân tử khoảng $5 \%$. Bên cạnh đó, kết quả tính bằng AFEM được so sánh với các phương pháp khác rất phù hợp. Điều đó cho thấy AFEM là một phương pháp đơn giản, nhanh chóng xác định đặc trưng đàn hồi cho các vật liệu cấu trúc na nô.

Tù khóa: phương pháp phần tử hữu hạn nguyên tử, đặc trưng đàn hồi, vật liệu na nô.

\section{GIỚI THIỆU}

Vật liệu na nô có cấu trúc lưới lục giác hay lưới tổ ong (Hình 1) đã được nghiên cứu trong những năm gần đây do những đặc tính ưu việt của chúng như làm chất gia cường cho vật liệu composite hay ứng dụng trong các thiết bị điện tử. Đầu tiên là năm 1991 khi ống các bon na nô được phát hiện trong phòng thí nghiệm [1].
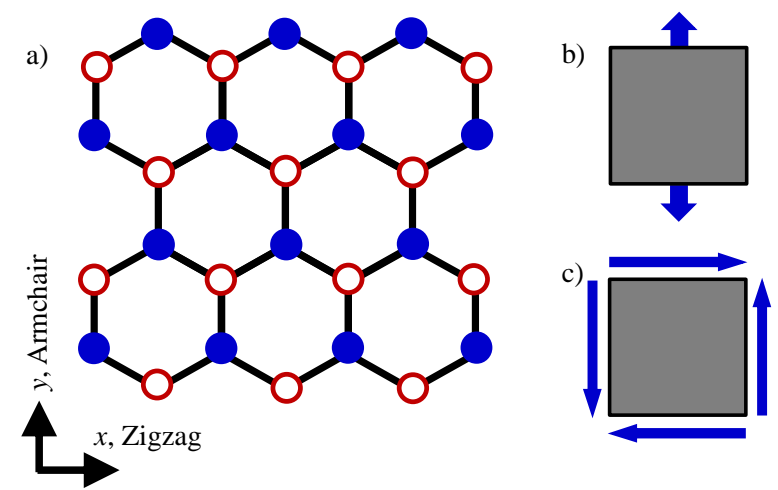

Hình 1. Mô hình a) cấu trúc tấm vật liệu bao gồm hai kiểu nguyên tử khác nhau,

b) thí nghiệm kéo đúng tâm, c) thí nghiệm trượt thuần túy. 
Đến năm 1993 ống các bon na nô đơn lớp (SWCNT) cũng được tìm ra [2]. Theo sau sự phát hiện ra graphen vào năm 2004, tới nay đã có thêm khoảng trên 20 loại vật liệu na nô có cấu trúc hình lục giác được dự đoán tồn tại trên lí thuyết. Trong đó có graphen [3] và BN [4 - 6] đã được tổng hợp trong thực tế.ưSau khi các vật liệu na nô trên được phát hiện, chúng cần được tính toán các đặc trưng cơ, lí, hóa để nhằm phục vụ cho sản xuất đại trà cũng như trước khi ứng dụng chúng trong thực tế. Trong khi tiến hành thực nghiệm ở thang na nô là rất khó khăn và đắt đỏ, việc mô phỏng số có vai trò quan trọng trong việc dự đoán các đặc trưng đàn hồi của các vật liệu na nô.

Phương pháp lí thuyết mật độ phiếm hàm (DFT), động lực học phân tử (MD) và tính toán tight-binding (TB) là những phương pháp mô phỏng tiên tiến cho kết quả chính xác, tuy nhiên chúng tốn nhiều thời gian tính toán.

Phương pháp phần tử hữu hạn trong nguyên tử (AFEM) đã được phát triển để tính toán cho các cấu trúc vật liệu na nô một cách hiệu quả [7 - 11]. Trong AFEM, nguyên tử được coi như các nút, chuyển vị của nguyên tử coi như chuyển vị của nút. Các phần tử trong AFEM được tạo ra để mô tả tương tác các thành phần thế năng giữa các nguyên tử. Thế năng của hệ được tính từ thế năng của mỗi phần tử. Đạo hàm bậc hai theo tọa độ các nút cho ra ma trận độ cứng của hệ. Tới đây, việc tính toán tương tự như trong phương pháp phần tử hữu hạn truyền thống (FEM).

Trong nghiên cứu này, AFEM được phát triển dựa trên hàm thế năng dạng hàm điều hòa để dự đoán đặc trưng đàn hồi của vật liệu có cấu trúc na nô. Ở biến dạng nhỏ, biến dạng dài và biến dạng góc của liên kết có thể được biểu diễn qua chuyển vị của mỗi nguyên tử và các hằng số lực. Hai kiểu phần tử được xây dựng trong bài báo này để mô tả cho biến dạng dài và biến dạng góc tương ứng. Ma trận được đưa ra dưới dạng hiển. Thông qua mô phỏng thí nghiệm kéo đơn trục và thí nghiệm trượt thuần túy (Hình 1 ), đặc trưng đàn hồi của ống các bon na nô đơn lớp, tấm graphen, ông và tấm $\mathrm{BN}$ đơn lớp được tính toán. Kết quả được so sánh với phương pháp $\mathrm{MD}$ cũng như các tài liệu khác để thấy được tính đúng đắn của nghiên cứu này.

\section{THIẾT LẬP MÔ HÌNH}

\subsection{Mô hình cơ học phân tử}

Thế năng tương tác giữa các nguyên tử có thể được biểu diễn bằng tổng thế năng tương tác liên kết và thế năng tương tác phi liên kết [12-14]:

$$
E=E_{r}+E_{\theta}+E_{\tau}+E_{\omega}+E_{v d w}+E_{e l}
$$

Xét trong biến dạng nhỏ, các hàm điều hòa thường được sử dụng để mô hình hóa cho thế năng biến dạng kéo $E_{r}$ và biến dạng góc $E_{\theta}$ như sau:

$$
\begin{gathered}
E_{r}=\frac{1}{2} \sum_{e=1}^{M} C_{r}^{e}\left(\delta t_{i j}^{e}\right)^{2}, \\
E_{\theta}=\frac{1}{2} \sum_{e=1}^{N} C_{i j k}^{e}\left(\delta \theta_{i j k}^{e}\right)^{2}
\end{gathered}
$$

trong đó: $\delta l_{i j}^{e}$ là biến dạng dài của liên kết thẳng thứ $e$ giữa hai nguyên tử $i$ và nguyên tử $j, \delta \theta_{i j k}^{e}$ là biến dạng góc giữa hai liên kết thẳng $i j$ và $j k$ (Hình 2). $M$ và $N$ tương ứng là tổng số biến dạng dài và biến dạng góc. $C_{r}^{e}$ và $C_{i j k}^{e}$ là hằng số lực tương ứng cho biến dạng dài và biến dạng góc. 
Với những tấm vật liệu cấu tạo bởi 2 loại nguyên tử (Hình 1a) sẽ có hai giá trị $C_{i j k}^{e}$ tương ứng phụ thuộc vào nguyên tử ở đỉnh của góc có biến dạng. Trong Hình $2 \mathrm{~b}$, nguyên tử $i$ và $k$ là cùng loại.

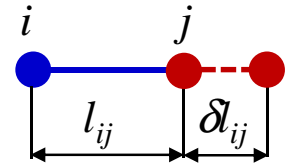

a)

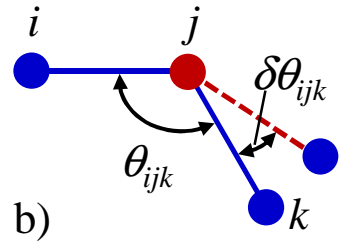

b)

Hình 2. Mô hình hai kiểu phần tử a) phần tử biến dạng thẳng $\mathrm{l}_{\mathrm{ij}}$ và biến dạng dài $\delta \mathrm{l}_{\mathrm{ij}}$;

b) phần tử biến dạng góc $\theta_{i j k}$ và biến dạng góc $\delta \theta_{i j k}$.

$E_{\tau}, E_{\omega}, E_{v d w}$, và $E_{e l}$ tương ứng là thế năng đến từ biến dạng xoắn, đảo liên kết, lực van der Waals và tương tác điện tử. Tất cả được bỏ qua trong giới hạn của nghiên cứu này.

\subsection{Mô hình phần tử hữu hạn}

\subsubsection{Xây dựng mô hình tấm và ống vật liệu na nô}

Tấm na nô đơn lớp được tạo ra từ hai thông số là chiều dài liên kết $\left(l_{0}\right)$ và góc liên kết $(\theta)$ ở vị trí cân bằng ban đầu (Hình 1 ).

Các đặc trưng cơ học của tấm phụ thuộc vào kích thước tấm (tức số lượng nguyên tử ít hay nhiều). Qua kết quả tính toán ở Bảng 1 , chúng tôi nhận thấy với vật liệu graphen từ tấm kích thước $10 \times 10 \mathrm{~nm}$ gồm 4032 nguyên tử trở đi giá trị mô đun đàn hồi theo hai phương không còn thay đổi nhiều trong khi khối lượng, thời gian tính toán tăng đáng kể. Do đó, tấm kích thước vuông gồm 4032 nguyên tử được chọn để mô phỏng cho graphen cũng tấm BN trong nghiên cứu này.

Bảng 1. Mô đun đàn hồi hai chiều $\mathrm{Y}_{\mathrm{s}}(\mathrm{N} / \mathrm{m})$ của graphen phụ thuộc vào kích thước tấm vuông.

\begin{tabular}{ccccccc}
\hline $\begin{array}{c}\text { Kích thước }(\mathrm{nm}) \\
\text { (Số nguyên tử) }\end{array}$ & $\approx 3 \times 3$ & $\approx 5 \times 5$ & $\approx 8 \times 8$ & $\approx 10 \times 10$ & $\approx 12 \times 12$ & $\approx 14 \times 14$ \\
\hline$Y_{\mathrm{s}}(N / m)$ phương armchair & 335,5 & 342,7 & 346,0 & 347,0 & 347,6 & 348,0 \\
$Y_{\mathrm{s}}(N / m)$ phương zigzag & 358,6 & 355,0 & 353,3 & 352,6 & 352,2 & 352,0 \\
\hline
\end{tabular}

Mô hình ống vật liệu na nô được tạo ra bằng cách cuộn tấm vật liệu theo phương véc tơ $\vec{C}_{h}$. Khi $\vec{C}_{h}$ trùng với phương zigzag (phương $x$ ) ta có ống dạng zigzag (n, 0$), \vec{C}_{h}$ trùng với phương armchair (phương $y$ ) ta có ống dạng armchair(n,n).

Xét tấm vật liệu na nô gồm $N$ nguyên tử (nút). Gọi $\left(x_{i}, y_{i}, z_{i}\right)$ là tọa độ trong không gian của nguyên tử thứ $i$. Trong đó phương trục $z$ vuông góc với mặt phẳng tấm. Vị trí của nguyên tử $i$ sau khi cuộn tấm thành ống là $\left(X_{i}, Y_{i}, Z_{i}\right)$ sẽ được xác định qua biểu thức sau:

Khi $\vec{C}_{h}$ trùng với phương zigzag: 


$$
X_{i}=R \sin \left(x_{i} / R\right) ; Y_{i}=y_{i} ; Z_{i}=R\left[\cos \left(x_{i} / R\right)-1\right]
$$

Khi $\vec{C}_{h}$ trùng với phương armchair:

$$
X_{i}=x_{i} ; Y_{i}=R \sin \left(y_{i} / R\right) ; Z_{i}=R\left[\cos \left(y_{i} / R\right)-1\right]
$$

trong phương trình (4) và (5), bán kính ống $R=\left|\vec{C}_{h}\right| / 2 \pi$.

Qua kết quả tính toán ở Bảng 2 cho thấy mô đun đàn hồi của SWCNT phụ thuộc khá nhiều vào tỉ lệ chiều dài trên đường kính ống $(\mathrm{L} / \mathrm{d})$. Khi chiều dài đủ lớn so với đường kính thì giá trị ít thay đổi hơn. Cụ thể với vật liệu $\mathrm{SWCNT}$, từ giá trị $\mathrm{L} \approx 15 \mathrm{~d}$ trở đi, mô đun đàn hồi đã khá ổn định. Do đó, tỉ lệ $\mathrm{L} \approx 15 \mathrm{~d}$ được chọn để xây dựng mô hình $\mathrm{SWCNT}$ cũng như cho ống $\mathrm{BN}$.

Bảng 2. Mô đun đàn hồi hai chiều $\mathrm{Y}_{\mathrm{s}}(\mathrm{N} / \mathrm{m})$ của $\mathrm{SWCNT}$ phụ thuộc vào tỉ lệ $\mathrm{L} / \mathrm{d}$.

\begin{tabular}{cccccc}
\hline Chỉ số, đường kính ống & $\mathrm{L} \approx 5 \mathrm{~d}$ & $\mathrm{~L} \approx 10 \mathrm{~d}$ & $\mathrm{~L} \approx 15 \mathrm{~d}$ & $\mathrm{~L} \approx 20 \mathrm{~d}$ & $\mathrm{~L} \approx 22 \mathrm{~d}$ \\
\hline$(10,0) ; \mathrm{d}=0,783 \mathrm{~nm}$ & 337,7 & 339,5 & 340,1 & 340,4 & 340,5 \\
$(10,10) ; \mathrm{d}=1,356 \mathrm{~nm}$ & 347,6 & 348,2 & 348,4 & 348,5 & 348,5 \\
\hline
\end{tabular}

\subsubsection{Thiết lập ma trận độ cứng phần tử}

Ta có thế năng biến dạng của một phần tử hai nút (hình 2a):

$$
E_{r}^{e}=\frac{1}{2} C_{r}^{e}\left(\delta l_{j i}^{e}\right)^{2}
$$

Xét tương tác giữa hai nguyên tử $i$ và $j$. Khoảng cách giữa chúng, $l_{j i}^{e}$, có thể được biểu diễn thông qua tọa độ $\left(x_{\mathrm{i}}, y_{\mathrm{i}}, z_{\mathrm{i}}\right)$ của nguyên tử $i$ và tọa độ $\left(x_{\mathrm{j}}, y_{\mathrm{j}}, z_{\mathrm{j}}\right)$ của nguyên tử $j$ như sau:

$$
\left(l_{j i}^{e}\right)^{2}=\left(x_{i}-x_{j}\right)^{2}+\left(y_{y}-y_{j}\right)^{2}+\left(z_{i}-z_{j}\right)^{2}
$$

Đạo hàm hai vế phương trình (7), ta có:

$$
l_{j i}^{e} \delta l_{j i}^{e}=\left(x_{i}-x_{j}\right)\left(\delta x_{i}-\delta x_{j}\right)+\left(y_{i}-y_{j}\right)\left(\delta y_{i}-\delta y_{j}\right)+\left(z_{i}-z_{j}\right)\left(\delta z_{i}-\delta z_{j}\right)
$$

Đặt $\delta x_{i}=q_{1}, \delta y_{i}=q_{2}, \delta z_{i}=q_{3}$, và $\delta x_{j}=q_{4}, \delta y_{j}=q_{5}$ và $\delta z_{j}=q_{6}$ là các chuyển vị của nguyên tử $i$ và $j$ theo các trục $x, y$, và $z$ tương tứng. Phương trình (8) trở thành:

$$
\delta l_{j i}^{e}=\frac{1}{l_{j i}^{e}}\left[x_{i j}\left(q_{1}-q_{4}\right)+y_{i j}\left(q_{2}-q_{5}\right)+z_{i j}\left(q_{3}-q_{6}\right)\right]
$$

Trong đó:

$$
x_{i j}=x_{i}-x_{j}, \quad y_{i j}=y_{i}-y_{j}, \quad z_{i j}=z_{i}-z_{j}
$$

ở trạng thái chưa biến dạng, ta có $l_{j i}^{e}=l_{0}$, với $l_{0}$ là chiều dài của liên kết thẳng ở trạng thái cân bằng ban đầu.

Thay phương trình (9) vào phương trình (6) ta có: 


$$
E_{r}^{e}=\frac{C_{r}^{e}}{2\left(l_{0}\right)^{2}}\left[x_{i j}\left(q_{1}-q_{4}\right)+y_{i j}\left(q_{2}-q_{5}\right)+z_{i j}\left(q_{3}-q_{6}\right)\right]^{2},
$$

trong đó $\mathbf{q}^{r}=\left\{q_{1}, q_{2}, \ldots, q_{6}\right\}^{T}$ là véc tơ chuyển vị nút của phần tử ba nút.

Đạo hàm bậc hai của hàm thế năng được xác định từ phương trình (11) theo chuyển vị nút cho ta được ma trận độ cứng của phần tử hai nút:

$$
k_{m p}^{r}=\frac{\partial E_{r}^{e}}{\partial q_{m} \partial q_{p}}, \quad m, p=1,2 \ldots 6
$$

Lập luận tương tự, thế năng của phần tử ba nút (hình 2b) có dạng như sau:

$$
E_{\theta}^{e}=\frac{1}{2} C_{i j k}^{e}\left(\delta \theta_{i j k}^{e}\right)^{2}
$$

trong đó góc $\theta_{i j k}$ giữa hai véc tơ $\mathbf{l}_{\mathrm{ji}}$ và $\mathbf{l}_{\mathrm{jk}}$ có thể được tính từ biểu thức sau:

$$
l_{j i} l_{j k} \cos \theta_{i j k}=x_{i j} x_{k j}+y_{i j} y_{k j}+z_{i j} z_{k j},
$$

trong đó $x_{i j}, y_{i j}, z_{i j}$ và $x_{k j}, y_{k j}, z_{k j}$ ký hiệu tương tự như (10):

Đạo hàm hai vế của phương trình (14) ta có:

$\delta \theta \sin \theta_{i j k}=\left(\frac{\delta l_{j i}}{l_{j i}}+\frac{\delta l_{j k}}{l_{j k}}\right) \cos \theta_{i j k}-\left(\frac{\delta x_{i j}}{l_{j i}} \frac{x_{k j}}{l_{j k}}+\frac{\delta y_{i j}}{l_{j i}} \frac{y_{k j}}{l_{j k}}+\frac{\delta z_{i j}}{l_{j i}} \frac{z_{k j}}{l_{j k}}\right)-\left(\frac{x_{i j}}{l_{j i}} \frac{\delta x_{k j}}{l_{j k}}+\frac{y_{i j}}{l_{j i}} \frac{\delta y_{k j}}{l_{j k}}+\frac{z_{i j}}{l_{j i}} \frac{\delta z_{k j}}{l_{j k}}\right)$

Chú ý rằng:

$\delta x_{i j}=q_{1}-q_{4} ; \delta y_{i j}=q_{2}-q_{5} ; \delta z_{i j}=q_{3}-q_{6} ; \delta x_{k j}=q_{7}-q_{4} ; \delta y_{k j}=q_{8}-q_{5} ; \delta z_{k j}=q_{9}-q_{6}$

trong đó $q_{7}, q_{8}$, và $q_{9}$ là chuyển vị của nguyên tử $k$ theo các phương $x, y$, và $z$ tương ứng.

Đối với cấu trúc tấm hình lục giác ở điều kiện chưa biến dạng ban đầu, ta có $l_{j i}=l_{j k}=l_{0}$ và $\theta_{i j k}=120^{\circ}$.

Chú ý đến phương trình (9), từ phương trình (15) cho ta:

$\delta \theta_{i j k}^{e}=\frac{1}{l_{0}^{2} \sin \theta}\left[\begin{array}{l}\left(x_{i j} \cos \theta+x_{j k}\right)\left(q_{1}-q_{4}\right)+\left(y_{i j} \cos \theta+y_{j k}\right)\left(q_{2}-q_{5}\right)+\left(z_{i j} \cos \theta+z_{j k}\right)\left(q_{3}-q_{6}\right) \\ +\left(x_{j k} \cos \theta+x_{i j}\right)\left(q_{4}-q_{7}\right)+\left(y_{j k} \cos \theta+y_{i j}\right)\left(q_{5}-q_{8}\right)+\left(z_{j k} \cos \theta+z_{i j}\right)\left(q_{6}-q_{9}\right)\end{array}\right]$

Thay phương trình (17) vào phương trình (13) ta có:

$$
E_{\theta}^{e}=\frac{C_{i j k}^{e}}{2\left(l_{0}^{2} \sin \theta\right)^{2}}\left[\begin{array}{l}
\left(x_{i j} \cos \theta+x_{j k}\right)\left(q_{1}-q_{4}\right)+\left(y_{i j} \cos \theta+y_{j k}\right)\left(q_{2}-q_{5}\right)+\left(z_{i j} \cos \theta+z_{j k}\right)\left(q_{3}-q_{6}\right) \\
+\left(x_{j k} \cos \theta+x_{i j}\right)\left(q_{4}-q_{7}\right)+\left(y_{j k} \cos \theta+y_{i j}\right)\left(q_{5}-q_{8}\right)+\left(z_{j k} \cos \theta+z_{i j}\right)\left(q_{6}-q_{9}\right)
\end{array}\right]^{2}
$$

Ma trận độ cứng $\mathbf{k}^{\theta}$ của phần tử 3 nút biến dạng góc được xác định như sau:

$$
k_{m p}^{\theta}=\frac{\partial E_{\theta}^{e}}{\partial q_{m} \partial q_{p}}, \quad m, p=1,2 \ldots 9 .
$$

\subsubsection{Thiết lập và giải hệ phuơng trình đặc trung}


Về bản chất AFEM hoàn toàn khác so với FEM truyền thống. Về hình thức thì AFEM lại có những điểm giống FEM truyền thống ở điểm sau:

i) Ma trận độ cứng tổng thể $\mathbf{K}$, véc tơ chuyển vị nút tổng thể $\mathbf{Q}$ và véc tơ lực nút tổng thể $\mathbf{F}$ của hệ được ghép nối tương ứng từ ma trận độ cứng, véc tơ chuyển vị và véc tơ lực nút của các phần tử.

ii) véc tơ chuyển vị nút và véc tơ lực nút nhận được từ điều kiện biên tương ứng sau khi giải phương trình đặc trưng:

$$
\mathbf{K} . \mathbf{Q}=\mathbf{F} \text {. }
$$

Việc thiết lập và giải phương trình (20) được lập trình trên phần mềm Matlab. Bài toán kéo đúng tâm đơn trục và bài toán trượt thuần túy (xem Hình 1) được mô phỏng để tìm đặc trưng đàn hồi của các tấm vật liệu graphen, $\mathrm{BN}$, ống $\mathrm{BN}$ và $\mathrm{SWCNT}$. Thông số của các vật liệu được đưa ra trong Bảng 3 .

Bảng 3. Các hằng số lực và chiều dài liên kết ban đầu của liên kết $\mathrm{C}-\mathrm{C}$ và $\mathrm{B}-\mathrm{N}$ ở nhiệt độ $0 \mathrm{~K}$.

\begin{tabular}{lcccccc}
\hline \multicolumn{1}{c}{ Vật liệu } & $\begin{array}{c}\text { Nguyên } \\
\text { tử 1 }\end{array}$ & $\begin{array}{c}\text { Nguyên } \\
\text { tử 2 }\end{array}$ & $\begin{array}{c}C_{r}^{e}, \\
\mathrm{kcal} /\left(\mathrm{mol}^{e} \AA^{2}\right)\end{array}$ & $\begin{array}{c}C_{121}^{e}, \\
\mathrm{kcal} /\left(\mathrm{mol}_{\mathrm{rad}}{ }^{2}\right)\end{array}$ & $\begin{array}{c}C_{212}^{e}, \\
\mathrm{kcal} /\left(\mathrm{mol}_{\mathrm{rad}}{ }^{2}\right)\end{array}$ & $\begin{array}{c}l_{0} \\
(\AA)\end{array}$ \\
\hline Graphen, SWCNT & $\mathrm{C}$ & $\mathrm{C}$ & 1051 & 195 & 195 & 1,42 \\
Ống và tấm BN & $\mathrm{B}$ & $\mathrm{N}$ & 856 & 95 & 191 & 1,45 \\
\hline
\end{tabular}

Trong đó chiều dài liên kết ban đầu $l_{0}$ được lấy từ tài liệu tham khảo [15]; các hằng số lực của liên kết $\mathrm{C}-\mathrm{C}$ được tham khảo từ [16], của liên kết $\mathrm{B}-\mathrm{N}$ tham khảo từ [17]. như sau:

Mô đun đàn hồi $Y$ và mô đun trượt $G$ được tính dựa trên biến dạng và ứng suất tương ứng

$$
Y=\frac{\sigma}{\varepsilon} ; G=\frac{\tau}{\gamma}
$$

trong đó $\sigma$ và $\varepsilon$ là ứng suất pháp và biến dạng dài trong thí nghiệm kéo đúng tâm; $\tau$ và $\gamma$ là ứng suất tiếp và biến dạng góc trong thí nghiệm trượt thuần túy của tấm.

Gọi $t$ là độ dày của tấm vật liệu. Ta có mô đun đàn hồi hai chiều, $Y_{\mathrm{s}}$, và mô đun đàn hồi trượt hai chiều, $G_{\mathrm{s}}$, được xác định như sau:

$$
Y_{\mathrm{s}}=Y t \text { và } G_{\mathrm{s}}=G t .
$$

\section{KẾT QUẢ VÀ THẢO LUẬN}

\subsection{Kết quả của mô hình tấm}

Kết quả được đưa ra trong Bảng 4 cho thấy rằng mô đun đàn hồi và mô đun đàn hồi trượt tính bởi phương pháp AFEM cho kết quả thấp hơn khoảng $5 \%$ so với kết quả tính được từ phương pháp MD. Trong khi đó, hệ số poisson có được từ phương pháp AFEM tăng nhẹ so với kết quả tính được từ phương pháp MD. Và trong cùng một loại vật liệu, hệ số poisson theo phương zigzag cao hơn so với phương armchair. Tuy nhiên, sai khác đó chỉ khoảng 0,01 nên có thể bỏ qua. Sai lệnh kết quả hệ số poisson tính bằng $\mathrm{AFEM}$ so với kết quả tính bằng $\mathrm{MD}$ trong Bảng 4 là rất nhỏ, khoảng $0,5 \%$. Nói chung, các kêt quả tính toán bằng phương pháp AFEM là 
rất gần với các kết quả tính bằng mô phỏng $\mathrm{MD}$. Điều đó cho thấy tính đúng đắn của phương pháp AFEM được đề xuất trong nghiên cứu này.

Bên cạnh đó, các kết quả tính toán đặc trưng đàn hồi của vật liệu sử dụng AFEM cũng được so sánh với các kết quả tính toán bằng các phương pháp khác được liệt kê trong bảng 4 . Sử dụng phương pháp AFEM tính ra mô đun đàn hồi hai chiều của graphen là $353 \mathrm{~N} / \mathrm{m}$ (phương zigzag) và $347 \mathrm{~N} / \mathrm{m}$ (phương armchair); mô đun đàn hồi trượt là $152 \mathrm{~N} / \mathrm{m}$ và hệ số poisson xấp xỉ 0,18. Các kết quả đó rất gần với các kết quả của các tác giả khác như: Yakobson [18] sử dụng mô hình vỏ mỏng dựa trên hàm thế Tersoff-Brenner, Popov và cộng sự [19] sử dụng mô hình động lực học tinh thể, Kudin và cộng sự [20] sử dụng mô hình vỏ dựa trên tính toán DFT, cũng như Sahin và cộng sự trong các tài liệu [15]. Và kết quả thu được từ AFEM cũng rất sát với kết quả đo từ thực nghiệm [21 - 23], khi độ dày tấm được giả thiết $t=3,35 \AA$ Å.

Bảng 4. Đặc trưng đàn hồi của tấm graphen và tấm $\mathrm{BN}$ đơn lớp được nghiên cứu và so sánh với các kết quả của các nghiên cứu khác.

\begin{tabular}{|c|c|c|c|c|}
\hline Vật liệu & $\begin{array}{l}\text { Phương pháp nghiên cứu, tác } \\
\text { giả }\end{array}$ & $\begin{array}{c}\text { Mô đun đàn hồi } \\
\text { hai chiều } \mathrm{E}_{\mathrm{s}}(N / m)\end{array}$ & $\begin{array}{l}\text { Mô đun trượt hai } \\
\text { chiều } \mathrm{G}_{\mathrm{s}}(\mathrm{N} / \mathrm{m})\end{array}$ & $\begin{array}{l}\text { Hệ số } \\
\text { Poisson }\end{array}$ \\
\hline \multirow[t]{10}{*}{ Graphen } & AFEM & 353 (zig) & 152 & 0,179 (zig) \\
\hline & & 347 (arm) & & $0,175(\mathrm{arm})$ \\
\hline & MD, Le and Nguyen 2014 [24] & $\begin{array}{l}364 \text { (zig) } \\
352 \text { (arm) }\end{array}$ & 158 & $0,15-0,17$ \\
\hline & $\begin{array}{l}\text { DFT \& mô hình vỏ, Kudin et } \\
\text { al. (2001) [20] }\end{array}$ & 345 & 150 & 0,149 \\
\hline & DFT, Sahin et al. (2009) [15] & 335 & & 0,16 \\
\hline & $\begin{array}{l}\text { Mô hình vỏ với hàm thế } \\
\text { Tersoff-Brenner, Yakobson et } \\
\text { al. (1996) [18] }\end{array}$ & 360 & & 0,19 \\
\hline & $\begin{array}{l}\text { Mô hình động lực học tinh thể, } \\
\text { Popov et al. (2000) [19] }\end{array}$ & $\begin{array}{l}1002 \text { GPa } \\
\text { (mô đun đàn hồi) }\end{array}$ & $\begin{array}{l}414 \mathrm{GPa} \\
\text { (mô đun trượt) }\end{array}$ & 0,21 \\
\hline & $\begin{array}{l}\text { Thí nghiệm tĩnh và cộng hưởng } \\
\text { âm, siêu âm, Blakslee et al. } \\
\text { (1970) [21] }\end{array}$ & $\begin{array}{l}1029 \text { GPa } \\
\text { (mô đun đàn hồi) }\end{array}$ & $\begin{array}{l}440 \mathrm{GPa} \\
\text { (mô đun trượt) }\end{array}$ & 0,17 \\
\hline & $\begin{array}{l}\text { Tán xạ tia X không đàn hồi, } \\
\text { Bosak et al. (2007) [22] }\end{array}$ & $\begin{array}{l}1092 \text { GPa } \\
\text { (mô đun đàn hồi) }\end{array}$ & $\begin{array}{l}485 \mathrm{GPa} \\
\text { (mô đun trượt) }\end{array}$ & \\
\hline & $\begin{array}{l}\text { Thực nghiệm, Lee et al. (2008) } \\
\text { [23] }\end{array}$ & $340 \pm 50$ & & \\
\hline \multirow[t]{7}{*}{$\mathrm{BN}$} & AFEM & 271 (zig) & 114 & 0,226 (zig) \\
\hline & & $266(\operatorname{arm})$ & & 0,222 (arm) \\
\hline & MD, Le and Nguyen 2014 [24] & $\begin{array}{l}280(\mathrm{zig}) \\
271 \text { (arm) }\end{array}$ & 117 & $0,19-0,22$ \\
\hline & $\begin{array}{l}\text { DFT \& mô hình vỏ, Kudin et } \\
\text { al. (2001) [20] }\end{array}$ & 271 & 112 & 0,211 \\
\hline & DFT, Sahin et al. (2009) [15] & 267 & & 0.21 \\
\hline & $\begin{array}{l}\text { DFT \& phương trình cân bằng, } \\
\text { Andrew et al. (2012) [25] }\end{array}$ & 275.8 & & \\
\hline & $\begin{array}{l}\text { Tán xạ tia X, Bosak et al. } \\
\text { (2006) [26] }\end{array}$ & $\begin{array}{l}776 \text { GPa } \\
\text { (mô đun đàn hồi) }\end{array}$ & $\begin{array}{l}321 \mathrm{GPa} \\
\text { (mô đun trượt) }\end{array}$ & 0,21 \\
\hline
\end{tabular}


Đặc trưng đàn hồi của vật liệu BN tính bởi AFEM: mô đun đàn hồi là $271 \mathrm{~N} / \mathrm{m}$ (phương zigzag) và $266 \mathrm{~N} / \mathrm{m}$ (phương armchair); mô đun đàn hồi trượt là $114 \mathrm{~N} / \mathrm{m}$ và hệ số Poisson xấp xỉ 0,22 . Những giá trị đặc trưng đàn hồi của $\mathrm{BN}$ nêu trên rất phù hợp với các giá trị tương ứng của các nghiên cứu trước đây bởi các phương pháp khác như sử dụng tính toán DFT [15, 20, 25], mô phỏng MD bởi Zhang và cộng sự [27]. Với giải thiết độ dày tấm $t=3,35 \AA$ A, mô đun đàn hồi và mô đun đàn hồi trượt của $\mathrm{BN}$ tính bằng $\mathrm{AFEM}$ rất gần với giá trị thực nghiệm được đo bởi Bosak và cộng sự [26].

\subsection{Kết quả của mô hình ống}

Qua thí nghiệm kéo theo phương hướng trục của ống, giá trị mô đun đàn hồi của các ống vật liệu được đưa ra ở Bảng 5 .

Bảng 5. Mô đun đàn hồi hai chiều $\mathrm{Y}_{\mathrm{s}}(\mathrm{N} / \mathrm{m})$ của $\mathrm{SWCNT}$ và ống $\mathrm{BN}$ đơn lớp theo đường kính ống, $\mathrm{d}$. Tỉ lệ chiều dài trên đường kính cố định $\mathrm{L} / \mathrm{d} \approx 15$.

\begin{tabular}{ccccc}
\hline \multirow{2}{*}{ Chỉ số } & \multicolumn{2}{c}{ Ông SWCNT } & \multicolumn{2}{c}{ Ông BN đơn lớp } \\
\cline { 2 - 5 }$(n, m)$ & $\mathrm{d}(\AA)$ & $\mathrm{Y}_{\mathrm{s}}(\mathrm{N} / \mathrm{m})$ & $\mathrm{d}(\AA)$ & $\mathrm{Y}_{\mathrm{s}}(\mathrm{N} / \mathrm{m})$ \\
\hline$(4,0)$ & 3,13 & 300,8 & 3,20 & 227,1 \\
$(5,0)$ & 3,91 & 315,7 & 4,00 & 238,5 \\
$(6,0)$ & 4,70 & 324,9 & 4,80 & 245,5 \\
$(7,0)$ & 5,48 & 330,9 & 5,60 & 250,2 \\
$(8,0)$ & 6,26 & 335,0 & 6,40 & 253,3 \\
$(9,0)$ & 7,05 & 337,9 & 7,20 & 255,6 \\
$(10,0)$ & 7,83 & 340,1 & 7,99 & 257,3 \\
$(15,0)$ & 11,74 & 345,4 & 11,99 & 261,4 \\
$(20,0)$ & 15,66 & 347,4 & 15,99 & 263,0 \\
$(3,3)$ & 4,07 & 333,3 & 4,15 & 252,2 \\
$(4,4)$ & 5,42 & 340,1 & 5,54 & 257,4 \\
$(5,5)$ & 6,78 & 343,5 & 6,92 & 260,0 \\
$(6,6)$ & 8,14 & 345,4 & 8,31 & 261,5 \\
$(7,7)$ & 9,49 & 346,6 & 9,69 & 262,4 \\
$(8,8)$ & 10,85 & 347,4 & 11,08 & 263,1 \\
$(9,9)$ & 12,20 & 348,0 & 12,46 & 263,5 \\
$(10,10)$ & 13,56 & 348,4 & 13,85 & 263,8 \\
$(12,12)$ & 16,27 & 348,9 & 16,62 & 264,2 \\
\hline & & & &
\end{tabular}

Kết quả cho thấy, ở cùng một đường kính, giá trị mô đun đàn hồi của ống dạng armchair lớn hơn so với ống dạng zigzag. Tuy vậy cả hai giá trị đều tăng khi đường kính ống tăng và tiến sát giá trị của vật liệu dạng tấm tương ứng khi đường kính ống đủ lớn. 


\section{KẾT LUẬN}

Trong nghiên cứu này, AFEM được đề xuất để tính các đặc trưng đàn hồi của các vật liệu lưới na nô lục giác. Thí nghiệm kéo đúng tâm và thí nghiệm trượt thuần túy được mô phỏng dựa trên AFEM để tính toán mô đun đàn hồi, mô đun đàn hồi trượt và hệ số Poisson của tấm graphen, tấm $\mathrm{BN}$ đơn lớp. Kết quả thu được rất gần với các kết quả tính toán bằng phương pháp khác như DFT, MD, ... Điều đó cho thấy tính đúng đắn của AFEM trong nghiên cứu này.

Với vật liệu dạng ống, qua bài toán kéo đúng tâm theo phương hướng trục, mô đun đàn hồi của ống được xác định. Nó tăng khi đường kính ống tăng và tiến tới giá trị của vật liệu dạng tấm tương ứng khi đường kính ống đủ lớn.

Mặc dù trong nghiên cứu này chỉ tập trung tính toán cho các vật liệu na nô cấu trúc dạng lục giác, AFEM hoàn toàn có thể áp dụng cho các cấu trúc vật liệu khác. Bên cạnh đó, do ma trận được tính ra dưới dạng hiển nên các phần tử của AFEM đề xuất trong nghiên cứu này có thể được đưa vào các phần mềm phần tử hữu hạn thương mại. So với các phương pháp mô phỏng ở thang na nô như $\mathrm{DFT}, \mathrm{MD}$, và tính toán $\mathrm{TB}, \mathrm{AFEM}$ là một phương pháp đơn giản, nhanh chóng tìm ra đặc trưng đàn hồi của các vật liệu cấu trúc na nô.

Lòi cảm ơn. Nghiên cứu này được tài trợ bởi Quỹ phát triển khoa học và công nghệ quốc gia (NAFOSTED) trong đề tài mã số: 107.02-2014.03.

\section{TÀI LIỆU THAM KHẢO}

1. Iijima S. - Helical microtubules of graphitic carbon, Nature 354 (1991) 56-58.

2. Iijima S. and Ichihashi T. - Single-shell carbon nanotubes of 1-nm diameter, Nature $\mathbf{3 6 3}$ (1993) 603-605.

3. Novoselov K. S., Geim A. K., Morozov S. V., Jiang D., Zhang Y., Dubonos S. V., Grigorieva I. V., and Firsov A. - Electric Field Effect in Atomically Thin Carbon Films, Science 306 (5696) (2004) 666-669.

4. Pacilé D., Meyer J. C., Girit Ç. Ö., and Zettl A. - The two-dimensional phase of boron nitride: Few-atomic-layer sheets and suspended membranes, Appl. Phys. Lett. 92 (13) (2008) 133107.

5. Shi Y., Hamsen C., Jia X., Kim K. K., Reina A., Hofmann M., Hsu A. L., Zhang K., Li H., Juang Z. Y., Dresselhaus M. S., Li L. J., and Kong J. - Synthesis of Few-Layer Hexagonal Boron Nitride Thin Film by Chemical Vapor Deposition, Nano Letters 10 (10) (2010) 4134-4139.

6. Song L., Ci L., Lu H., Sorokin P.B., Jin C., Ni J., Kvashnin A. G., Kvashnin D. G., Lou J., Yakobson B. I., and Ajayan P. M. - Large Scale Growth and Characterization of Atomic Hexagonal Boron Nitride Layers, Nano Letters 10 (8) (2010) 3209-15.

7. Nasdala L. and Ernst G. - Development of a 4-node finite element for the computation of nano-structured materials, Computational Materials Science 33 (2005) 443-458.

8. Wackerfuß J. - Molecular mechanics in the context of the finite element method, Int. J. Numer. Meth. Eng. 77 (2009) 969-997.

9. Liu B., Huang Y., Jiang H., Qu S., and Hwang K. C. - The atomic-scale finite element method, Comput. Methods Appl. Mech. Engrg. 193 (2004) 1849-1864. 
10. Wang Y., Zhang C., Zhou E., Sun C., Hinkley J., Gates T., and Su J. - Atomistic finite elements applicable to solid polymers, Computational Materials Science 36 (2006) 292-302.

11. Nasdala L., Kempe A., and Rolfes R. - The Molecular Dynamic Finite Element Method, CMC 19 (1) (2010) 57-104.

12. Burkert U. and Allinger N. L. - Molecular Mechanics: American Chemical Society Publication, 1982.

13. Leach A. R. - Molecular Modelling Principles and Applications. 2 ed: Prentice Hall, 2001.

14. Li C. and Chou T. W. - A structural mechanics approach for the analysis of carbon nanotubes, International Journal of Solids and Structures 40 (2003) 2487-2499.

15. Sahin H., Cahangirov S., Topsakal M., Bekaroglu E., Akturk E., Senger R. T., and Ciraci S. - Monolayer honeycomb structures of group-IV elements and III-V binary compounds: First-principles calculations, Phys. Rev. B 80 (15) (2009) 155453.

16. Berinskii I. E. and Borodich F. M. - Elastic in-plane properties of 2D linearized models of graphene, Mechanics of Materials 62 (2013) 60-68.

17. Jiang L. and Guo W. - A molecular mechanics study on size-dependent elastic properties of single-walled boron nitride nanotubes, Journal of the Mechanics and Physics of Solids 59 (6) (2011) 1204-1213.

18. Yakobson B. I., Brabec C. J., and Bernholc J. - Nanomechanics of Carbon Tubes: Instabilities beyond Linear Response, Phys. Rev. Lett. 76 (14) (1996) 2511-2514.

19. Popov V. N., Van Doren V. E., and Balkanski M. - Elastic properties of single-walled carbon nanotubes, Physical Review B 61 (2000) 3078-3084.

20. Kudin K. N., Scuseria G. E., and Yakobson B. I. - C2F, BN, and C nanoshell elasticity from ab initio computations, Phys. Rev. B 64 (23) (2001) 235406.

21. Blakslee O. L., Proctor D. G., Seldin E. J., Spence G. B., and Weng T. - Elastic constants of compression-annealed pyrolytic graphite, Journal of Applied Physics 41 (1970) 3373-3382.

22. Bosak A., Krisch M., Mohr M., Maultzsch J., and Thomsen C. - Elasticity of singlecrystalline graphite: inelastic x-ray scattering study, Physical Review B 75 (15) (2007) 153408.

23. Lee C., Wei X., Kysar J. W., and Hone J. - Measurement of the Elastic Properties and Intrinsic Strength of Monolayer Graphene, Science 321 (2008) 385-388.

24. Le Minh Quy and Nguyen Danh Truong - Determination of elastic properties of hexagonal sheets by atomistic finite element analysis, J. Comput. Theor. Nanosci. (2014) (accept).

25. Andrew R. C., Mapasha R. E., Ukpong A. M., and Chetty N. - Mechanical properties of graphene and boronitrene, Phys. Rev. B 85 (12) (2012) 125428.

26. Bosak A., Serrano J., and Krisch M. - Elasticity of hexagonal boron nitride: Inelastic X-ray scattering measurements, Phys. Rev. B 73 (04) (2006) 041402R.

27. Zhang D. B., Akatyeva E., and Dumitrica T. - Helical BN and $\mathrm{ZnO}$ nanotubes with intrinsic twisting: An objective molecular dynamics study, Physical Review B 84 (11) (2011) 115431. 


\title{
ABSTRACT \\ DERTERMINATION OF ELASTIC PROPERTIES OF NANOSHEETS AND NANOTUBES BY ATOMISTIC FINITE ELEMENT METHOD
}

\author{
Nguyen Danh Truong ${ }^{*}$, Le Minh Quy \\ School of Mechanical Engineering, Hanoi University of Science and Technology, \\ No 1 Dai Co Viet, Hanoi. \\ "Email: Truong.nguyendanh@hust.edu.vn
}

An atomistic finite element method (AFEM) was applied to determine elastic properties of graphene and boron nitride (BN) monolayer sheets. Using the same force field parameters, deviations between results obtained by AFEM and those by molecular dynamics simulations appear within $5 \%$ for Young's and shear moduli. By comparing simulation results with available data in the literature for these 2 sheets, it may be concluded that the proposed AFEM is a simple and fast technique to analyze accurately elastic properties of nanostructured materials.

Keywords: Atomistic finite element method; elastic properties; nanomaterial. 